\title{
Desalinhamento cambial, regimes de acumulação e metas de inflação em um modelo pós-keynesiano de crescimento *
}

\author{
José Luis Oreiro ** \\ Laura Díaz Abramo *** \\ Pedro Garrido da Costa Lima ${ }^{\text {**** }}$
}

\begin{abstract}
Resumo
O presente artigo tem por objetivo fazer uma extensão do modelo Oreiro-Araújo, incorporando ao menos três elementos novos, a saber: a determinação da taxa de inflação com base em conflito distributivo entre salários e lucros, a introdução de uma regra de ajuste da taxa real de câmbio e a condução da política monetária em um contexto de metas inflacionárias. No equilíbrio de curto prazo verifica-se que a relação entre crescimento e distribuição, o assim chamado regime de acumulação, depende da relação entre a taxa real de câmbio e o valor ótimo dessa taxa. Quando a taxa real de câmbio está sobrevalorizada, ou seja, abaixo do valor ótimo, o regime de acumulação é profit-led. Quando a taxa real de câmbio se encontra subvalorizada, ou seja, acima do seu valor ótimo, então o regime de acumulação é do tipo wage-led. No equilíbrio de longo prazo verifica-se que um aumento da meta de inflação está associado a uma redução da taxa real de juros e a um aumento da taxa real de câmbio, o que resulta em uma elevação da taxa de acumulação de capital, apesar do aumento da taxa de inflação.
\end{abstract}

Palavras-chave: Crescimento econômico; Distribuição de renda; Regimes de acumulação; Desalinhamento cambial; Metas de inflação.

\section{Abstract \\ Exchange rate misalignment, accumulation regimes and inflation targeting in a post-Keynesian growth model}

The objective of the present article is to carry out an extension of the Oreiro-Araújo model, incorporating three novel elements to its structure: the theory of conflict inflation between wages and profits, the rule for exchange rate adjustments and the monetary policy under an inflation targeting regime. In the short-run equilibrium, the relation between growth and income distribution - the socalled accumulation regime - depends on the relation between the level of real exchange rate and the its optimum value. When the real exchange rate is overvalued - i.e. below the optimum value accumulation regime is profit-led. When the real exchange rate is undervalued - i.e. above the optimum value - accumulation regime is wage-led. In the long-run equilibrium, it can be shown that an increase

* Artigo recebido em 20 de janeiro de 2014 e aprovado em 2 de novembro de 2016. Os autores agradecem os comentários de um parecerista da desta revista, os quais foram muito úteis para a versão final do artigo. Eventuais falhas remanescentes são de nossa inteira responsabilidade.

** Professor do Instituto de Economia da Universidade Federal do Rio de Janeiro (IE/UFRJ) / Pesquisador nível IB do Conselho Nacional de Desenvolvimento Científico e Tecnológico (CNPq), Rio de Janeiro, RJ, Brasil. E-mail: jose.oreiro@ie.ufrj.br.

*** Mestre em Economia pela Universidade de Brasília ( $\underline{\mathrm{UnB}})$ e assessora no Ministério da Fazenda, Brasília, DF, Brasil. E-mail: lauradiazabramo@gmail.com.

**** Doutor em Economia pela UnB e Consultor Legislativo da Câmara dos Deputados, Brasília, DF, Brasil. E-mail: pedrogarrido@gmail.com. 
in target inflation is associated with a reduction in the real interest rate and with a real exchange rate depreciation, which induces an increase in the rate of capital accumulation, despite the increase in the rate of inflation.

Keywords: Economic growth; Income distribution; Accumulation regimes; Real exchange rate misalignment; Inflation targeting.

JEL E12, O11, 023.

\section{Introdução}

Nos últimos anos tem sido desenvolvida uma interessante literatura a respeito da relação entre câmbio real e crescimento econômico. O artigo seminal de Razin e Collins (1997) apontou para a existência de importantes não lineariedades na relação entre avariável desalinhamento cambial - definida como um desvio duradouro da taxa real de câmbio com respeito a algum valor de referência, determinado pelos "fundamentos" - e o crescimento do produto real para uma amostra de 93 países desenvolvidos e em desenvolvimento no período 1975-1993. Com efeito, os resultados empíricos mostraram que enquanto apenas sobrevalorizações muito intensas da taxa real de câmbio estão associadas com crescimento econômico mais lento no longoprazo, subvalorizações moderadas do câmbio real têm efeito positivo sobre o crescimento do produto interno bruto. Rodrik (2008), ao analisar as estratégias de desenvolvimento adotadas por um conjunto de países, notou que um fator importante para a ignição de um processo de crescimento sustentado do produto real é a manutenção de uma taxa real de câmbio depreciada e estável. De forma similar, Frenkel (2004) -ao analisar a performance do emprego e da taxa de crescimento da Argentina, Brasil, Chile e México - constatou que a manutenção de uma taxa real de câmbio competitiva e estável é a melhor contribuição que a política macroeconômica pode dar para o crescimento econômico de longoprazo. Para o caso brasileiro, Oreiro, Punzo e Aráujo (2012) apontaram para a existência de um efeito negativo e estatisticamente significativo do desalinhamento cambial sobre a taxa de crescimento do produto real no período 1994-2007.

A relação entre câmbio real e crescimento tem sido, contudo, negligenciada pela literatura pós-keynesiana de crescimento. No contexto dos assim chamados modelos de crescimento com restrição de balanço de pagamentos, desenvolvidos pioneiramente por Thirlwall (1979), a taxa de crescimento de equilíbrio de longo prazo depende da razão entre as elasticidades-renda das exportações e das importações multiplicada pela taxa de crescimento da renda do resto do mundo. Variações da taxa real de câmbio são supostas irrelevantes para o crescimento de longo prazo, quer pela constatação empírica de que as elasticidades-preço das exportações e das importações são baixas, de tal forma que o impacto de uma desvalorização real do câmbio sobre o ritmo de crescimento das exportações e das 
importações é reduzido; quer pelo fato de que os termos de troca não apresentam uma tendência sistemática à apreciação ou depreciação no longo prazo (Mccombie; Roberts, 2002, p. 92).

No contexto dos assim chamados modelos neokaleckianos de crescimento e distribuição de renda, o nível da taxa real de câmbio pode afetar o crescimento de longo prazo, devido ao impacto daquela variável sobre a distribuição funcional da renda. Com efeito, se prevalecer um regime de acumulação do tipo profit-led, uma desvalorização da taxa real de câmbio irá resultar em um aumento do grau de utilização da capacidade produtiva e da taxa de investimento ${ }^{1}$. Esse resultado se deve ao fato de que a desvalorização da taxa real de câmbio irá ocasionar uma redução do salário real, aumentando assim a margem de lucro das empresas, o que tem um efeito positivo sobre o investimento planejado pelas mesmas (Bhaduri; Marglin, 1990; Blecker, 2002). A queda do salário real irá, no entanto, reduzir a demanda de consumo devido às diferenças existentes entre a propensão a consumir dos trabalhadores e dos capitalistas. No entanto, se a sensibilidade do investimento às variações da margem de lucros for elevada e se a diferença entre as propensões a consumir entre capitalistas e trabalhadores for reduzida; então a queda da demanda de consumo induzida pela redução de salário real será mais do que compensada pelo aumento da demanda de investimento, ocasionando assim um aumento do grau de utilização da capacidade produtiva. Caso contrário, a redução do salário real produzida pela desvalorização da taxa de câmbio irá resultar em uma queda do grau de utilização da capacidade produtiva, o que tem efeitos negativos sobre o investimento e, consequentemente, sobre a taxa de crescimento do produto real devido ao assim chamado "efeito acelerador”. Nesse caso, a economia estará operando com um regime wage-led.

A relação entre desalinhamento cambial e regimes de acumulação foi analisada pioneiramente por Oreiro e Araujo (2013). Esses autores desenvolvem um modelo neokaleckiano de crescimento e distribuição, com base no modelo original de Bhaduri e Marglin, no qual a taxa desejada de acumulação de capital é uma função quadrática da taxa real de câmbio. Nesse contexto, demonstra-se a existência de uma

(1) Blecker (2010) distingue economias ditas "domesticamente" profit-led, nas quais o efeito positivo sobre o investimento de um aumento da participação dos lucros na renda é maior do que o efeito negativo sobre o consumo de tal variação, das economias "globalmente" profit-led, nas quais o efeito líquido positivo do aumento da participação dos lucros sobre a demanda agregada é decorrente do aumento das exportações líquidas, embora o efeito negativo sobre o consumo seja superior ao efeito positivo sobre o investimento, fazendo com que a relação entre a participação dos lucros na renda e a absorção doméstica seja negativa. Nesse contexto, estudos empíricos como os realizados por Hein e Voguel (2008) mostram que a propensão à ocorrência de regimes de demanda do tipo profit-led é tão maior quanto maior for o grau de abertura comercial da economia. No que se refere ao caso brasileiro, Araujo e Gala (2012) mostram que o regime de demanda é "globalmente" profit-led, pois embora a absorção doméstica reaja negativamente a um aumento da participação dos lucros na renda, ao se considerar as exportações líquidas, obtêm-se um regime de demanda claramente profit-led. 
taxa de câmbio ótima, a qual maximiza a taxa desejada de acumulação de capital. Se a taxa real de câmbio estiver acima desse valor ótimo, então o câmbio se encontra sobrevalorizado; caso contrário, o câmbio estará subvalorizado. Quando a taxa real de câmbio está sobrevalorizada, o regime de acumulação é do tipo profit-led, ou seja, um aumento da participação dos lucros na renda irá induzir tanto um aumento do grau de utilização da capacidade produtiva, como um aumento da taxa de acumulação de capital. Já quando a taxa de câmbio se encontra subvalorizada o regime de acumulação é do tipo wage-led.

O modelo originalmente desenvolvido por Oreiro e Araujo, contudo, não considerava os efeitos da política macroeconômica sobre a configuração de equilíbrio do sistema. De fato, não só a taxa real de câmbio era suposta uma variável exógena ao sistema; como, além disso, variações do câmbio real e, portanto, da distribuição funcional da renda, não se expressavam em variações da taxa de inflação. A inclusão da taxa de inflação na estrutura do modelo macrodinâmico é de fundamental importância para que se possa entender os possíveis obstáculos a obtenção da "taxa de câmbio ótima"; uma vez que a autoridade monetária pode ser levada a aumentar a taxa de juros de curtoprazo face a uma aceleração inflacionária que resulte da desvalorização da taxa de câmbio. Essa questão é particularmente importante caso a política monetária seja conduzida com base em um regime de metas de inflação. Nesse contexto, pode existir um trade-off entre inflação e desalinhamento cambial, ou seja, uma redução da sobre-valorização cambial pode ser possível apenas mediante a elevação da meta de inflação.

Isso posto, o presente artigo tem por objetivo fazer uma extensão do modelo Oreiro-Araujo, incorporando ao mesmo tres elementos novos, a saber: a determinação da taxa de inflação com base em conflito distributivo entre salários e lucros, a introdução de uma regra de ajuste da taxa real de câmbio e a condução da política monetária em um contexto de metas inflacionárias.

\section{Estrutura do modelo}

Consideremos uma economia aberta que produz um único bem homogêneo e onde há atividades governamentais. O crescimento da economia em questão depende do grau de utilização da capacidade produtiva, o qual é considerado uma variável endógena no modelo. Assim como Kaldor (1956), iremos supor que existem dois tipos de agentes, a saber, capitalistas e trabalhadores. A propensão a poupar dos capitalistas é maior do que zero, ao passo que os trabalhadores gastam toda a sua renda com bens de consumo.

As firmas, por sua vez, operam em uma estrutura de mercado oligopolizada, isto é, elas fixam seus preços com base em um mark-up sobre os custos variáveis de 
produção. Dado que o único insumo variável utilizado na produção é o trabalho e que a taxa de mark-up é igual para todas as firmas, temos a seguinte lei de formação de preços:

$$
P=(1+z) w b
$$

Onde: $P$ representa o preço dos bens, $z$ é a taxa de mark-up, $w$ é a taxa nominal de salários e $b$ é o requisito unitário de mãodeobra, representando a razão entre a quantidade de trabalhadores e o produto total.

Iremos admitir que o bem final produzido nesta economia é um substituto imperfeito dos bens finais produzidos no resto do mundo, de tal forma que a lei do preço único não se aplica. Esta ausência de paridade de poder de compra faz com que o poder de monopólio das firmas domésticas seja afetado pelo preço dos bens importados. Por conseguinte, a taxa de mark-up das firmas será uma função que depende da taxa de câmbio real. Neste contexto, uma desvalorização da taxa de câmbio real permite que as empresas domésticas aumentem seu mark-up, devido ao aumento do poder de monopólio:

$$
z=z_{0}+z_{1} \theta \text { (2), sendo } z_{1}>0
$$

A participação dos lucros na renda $(m)$, por sua vez, é determinada a nível microeconômico com base no mark-up fixado pelas firmas. Assim, temos que:

$$
m=\frac{z}{1+z}=\frac{z_{0}+z_{1} \theta}{1+z_{0}+z_{1} \theta}
$$

Diferenciando (3) com relação $a \theta$, obtemos:

$$
\frac{\partial m}{\partial \theta}=\frac{z_{1}}{\left(1+z_{0}+z_{1} \theta\right)^{2}}>0
$$

Dessa forma uma desvalorização da taxa de câmbio real provoca um aumento da participação dos lucros na renda.

Tal como em Oreiro e Araújo (2013), a taxa desejada de crescimento do estoque de capital é uma função que depende da participação dos lucros na renda, do grau de utilização da capacidade produtiva e da taxa real de câmbio real. A versão modificada neste artigo supõe, ademais, que a função de acumulação de capital também é afetada pela taxa real de juros.

Sabemos que uma taxa de câmbio real desvalorizada estimula o investimento, uma vez que ela permite um aumento do poder de monopólio das firmas domésticas e, consequentemente, um aumento da taxa de mark-up e da participação dos lucros na renda. No entanto, uma vez que parte dos bens de capital necessários para a produção é importado, temos que uma taxa de câmbio real excessivamente desvalorizada pode desencorajar as decisões de investimento, 
devido à elevação dos preços dos bens de capital vindos do exterior. Segue-se portanto que, a partir de certo valor crítico, o investimento torna-se uma função inversa da taxa de câmbio real. Sendo assim, iremos supor que o investimento em capital fixo é uma função não linear da taxa real de câmbio.

Com base nessas considerações temos que a taxa desejada de acumulação do estoque de capital é dada por:

$$
\frac{I}{K}=\alpha_{0}+\alpha_{1} m+\alpha_{2} u+\alpha_{3} \theta-\alpha_{4} \theta^{2}-\alpha_{5} r(5)^{23}
$$

Onde: $\frac{I}{K}$ representa o investimento desejado pelas firmas como fração do estoque de capital; $m$ é a participação dos lucros na renda; $u$ é o grau de utilização da capacidade produtiva; $\theta=\frac{e P^{*}}{P}$ é a taxa de câmbio real; $r$ é a taxa real de juros e $\alpha_{i}, i=1, \ldots 5$ são parâmetros positivos.

Diferenciando (5) em relação $\mathrm{a} \theta$, obtemos:

$$
\frac{\partial \frac{I}{K}}{\partial \theta}=\alpha_{1} m^{\prime}+\alpha_{3}-2 \theta \alpha_{4} \quad \text { onde: } m^{\prime}=\frac{\partial m}{\partial \theta}(6)
$$

Dada a equação acima, podemos encontrar o valor da taxa de câmbio real que maximiza a taxa de acumulação de capital da economia:

$$
\theta^{*}=\frac{\alpha_{1} m^{\prime}+\alpha_{3}}{2 \theta \alpha_{4}}
$$

Do exposto acima, definimos sobrevalorização da taxa de câmbio real como uma situação em que $\theta$ se encontra abaixo do valor que maximiza a taxa de acumulação de capital da economia $\left(\theta^{*}\right)$ e uma subvalorização da taxa de câmbio real como uma situação em que $\theta$ se encontra acima deste valor crítico.

(2) Deve-se observar que na equação acima estamos supondo que a taxa real de câmbio exerce uma influência separada e adicional à influência da participação dos lucros na renda sobre a decisão de investimento em capital fixo. A princípio tal especificação da função investimento poderia sugerir uma duplicação desnecessária de variáveis à medida que a participação dos lucros na renda depende, ela própria, da taxa real de câmbio. Contudo, optamos por essa especificação da função investimento com base na ideia de que as variações puramente autônomas da participação dos lucros na renda afetam o investimento. Com efeito, uma variação puramente autônoma na taxa de mark-up [um aumento em $z_{0}$ na equação (2)] irá resultar em uma redução do salário real e, portanto, em um aumento da participação dos lucros na renda que é autônomo com respeito à variação da taxa real de câmbio. Um aumento autônomo da participação dos lucros na renda pode induzir um nível maior de investimento à medida que estiver associado à geração de um fluxo maior de recursos próprios para a firma, reduzindo assim tanto o "risco do tomador" como o "risco do emprestador", diminuindo assim a taxa de retorno requerida para a realização de projetos de investimento.

(3) Uma estimação econométrica da equação (5) para o caso brasileiro no período 1994. T3 a 2008. T4 pode ser obtida em Oreiro e Araujo (2013). 
Figura 1

Acumulação de capital e taxa de câmbio real

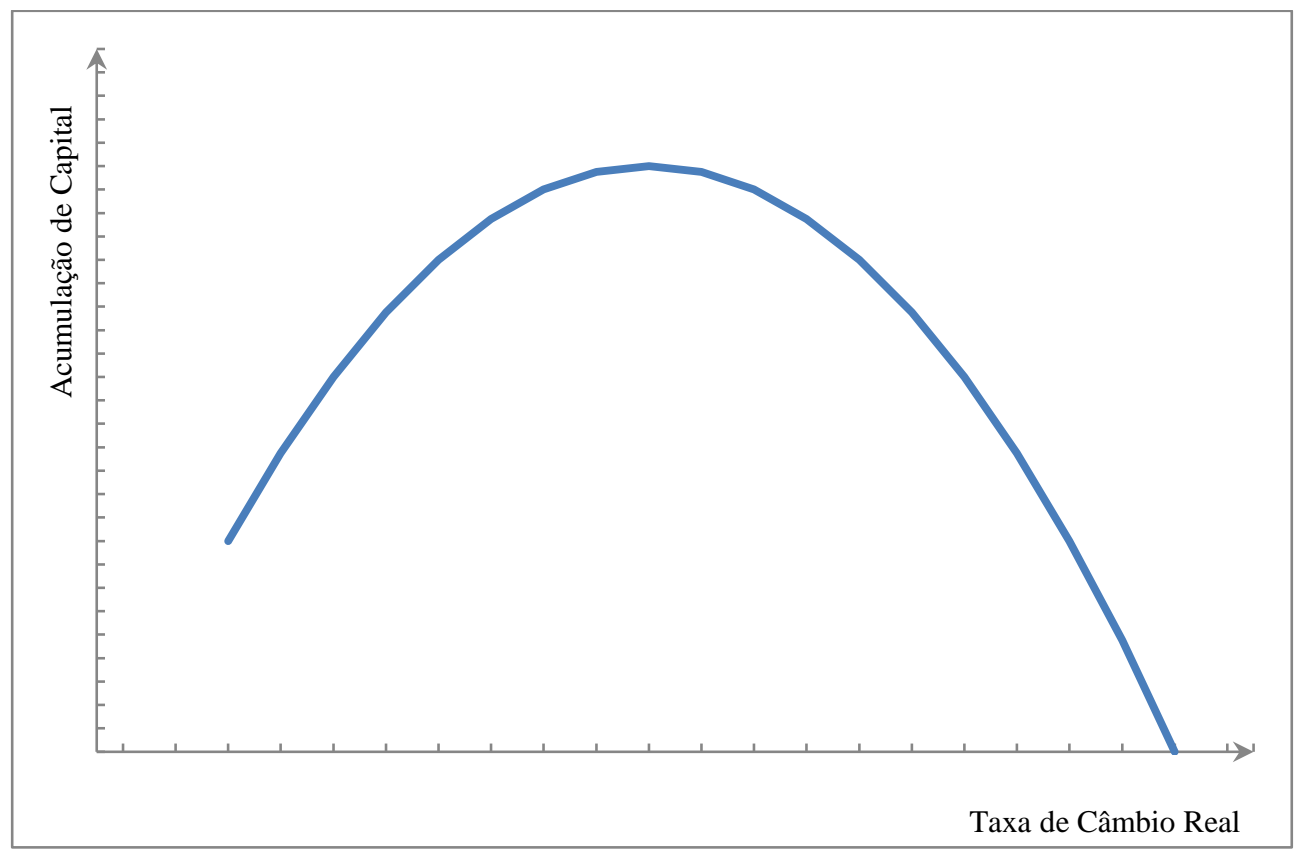

Tal como em Neves e Oreiro (2009), iremos supor que a propensão a consumir a partir dos salários é igual a zero, ao passo que a propensão a consumir a partir dos lucros é igual a $\left(1-s_{C}\right)$, de tal forma que a função consumo desta economia é dada pela expressão:

$$
\frac{C}{K}=(1-m) u+\left(1-s_{c}\right) m u
$$

Onde: $\frac{C}{K}$ representa o consumo como fração do estoque de capital; $(1-m) u$ é o consumo dos trabalhadores; $\left(1-s_{c}\right) m u$ é o consumo dos capitalistas e $s_{c}$ é a propensão a poupar dos capitalistas.

Os gastos do governo são normalizados como uma fração do estoque de capital e supostos constantes ao longo do tempo ${ }^{4}$ :

$$
\frac{G}{K}=\gamma
$$

Iremos supor que a função das exportações líquidas como fração do estoque de capital ${ }^{5}$ dada por:

(4) Por simplicidade iremos supor que a arrecadação de impostos é igual azero.

(5) Assumindo que a condição de Marshall-Lerner é válida, uma desvalorização da taxa de câmbio real gera um aumento das exportações líquidas. 


$$
\frac{E}{K}=\varepsilon_{0}+\varepsilon_{1} \theta-\varepsilon_{2} u
$$

Onde: $\varepsilon_{1}$ e $\varepsilon_{2}$ são constantes positivas e $\varepsilon_{0}=\varepsilon_{0,1}+\varepsilon_{0,2} u^{*}$, sendo $\varepsilon_{0,1}$ e $\varepsilon_{0,2}$ constantes positivas e $u^{*}$ é o grau de utilização da capacidade produtiva do "resto do mundo".

De acordo com Neves e Oreiro (2009), no curto prazo, as empresas podem estar dispostas a sacrificar uma parte de sua rentabilidade em prol de um maior market-share. Isto implica que a taxa de mark-up que prevalece em determinado ponto do tempo não necessariamente coincide com a taxa de mark-up que as empresas desejam obter no longo prazo. Se definirmos $z^{d}$ como essa taxa de markup desejada, então, $m^{d}$ representa a participação dos lucros na renda que é desejada pelos capitalistas:

$$
m^{d}=\eta_{0}+\eta_{1} u
$$

Onde $\eta_{0}, \eta_{1}$ são parâmetros positivos.

A equação acima nos mostra que a participação desejada dos lucros na renda depende positivamente do grau de utilização da capacidade produtiva. De fato, um maior nível de utilização da capacidade produtiva está associado a um maior poder de monopólio por parte das firmas, aumentando assim a taxa desejada de mark-up.

A inflação, por sua vez, resulta da tentativa das firmas em ajustar o mark-up efetivo ao mark-up desejado ${ }^{6}$. Sendo assim, temos que:

$$
\pi=\varphi\left(\eta_{0}+\eta_{1} u-m\right)
$$

Onde $\pi$ representa a taxa de inflação e $\varphi$ é um parâmetro positivo.

Como em Basilio e Oreiro (2008), iremos supor que o regime cambial prevalecente na economia é o regime de câmbio administrado, o qual opera em um contexto de mobilidade de capitais igual a zero ${ }^{7}$. Dessa forma, a autoridade monetária pode administrar a taxa nominal de câmbio de forma a garantir que a

(6) Neste modelo iremos assumir que os trabalhadores não possuem poder de mercado, de forma que eles não são capazes de determinar o salário real, mas apenas o salário nominal. Além disso, iremos assumir que a inflação esperada pelos trabalhadores é igual a zero, de maneira que os salários nominais são mantidos constantes ao longo do tempo. Dessa forma, a regra de formação de preços com base em mark-up: $P_{t}=\left(1+z_{t}\right) W b$ implica que: $\frac{P_{t}}{P_{t-1}}=\left(1+\pi_{t}\right)=\frac{\left(1+z_{t}\right)}{\left(1+z_{t-1}\right)}$, onde $: \pi_{t}$ é a taxa de inflação no período t. Para valores baixos de $\pi_{t}, z_{t}$ e $z_{t-1}$ essa expressão pode ser aproximada por (cf. Blanchard, 2007, p.276): $\pi_{t}=z_{t}-z_{t-1}$, ou seja, a taxa de inflação no período t será igual a variação da taxa de mark-up entre t-1 e t. Por fim, iremos supor que o ajuste do mark-up efetivo ao mark-up desejado é feito de forma gradual pelas firmas de maneira que: $z_{t}-z_{t-1}=\varphi\left(m^{d}-m\right)$. Dessa forma, temos que: $\pi_{t}=z_{t}-z_{t-1}=\varphi\left(m^{d}-m\right)$.

(7) A hipótese de mobilidade de capitais igual à zero significa que a conta de capitais da economia em consideração está fechada, de tal maneira que não é possível a realização de operações de arbitragem entre títulos denominados em moeda doméstica e títulos denominados em moeda estrangeira. Dessa forma, a autoridade monetária não se defronta com dificuldades maiores para administrar câmbio e juros ao mesmo tempo e de forma independente entre si. 
trajetória temporal da taxa de câmbio real seja compatível com o equilíbrio externo da economia no longo prazo ${ }^{8}$. Para tanto, a taxa de variação do câmbio nominal fixada pelo Banco Central será igual à diferença entre a inflação doméstica e a inflação internacional acrescida da variação do câmbio real que é desejada pela autoridade monetária para fazer um ajuste gradual entre o saldo efetivo de exportações líquidas $\left(\frac{E}{K}\right)$ e o saldo de exportações líquidas desejado pelo Banco Central $\left(\frac{E}{K}\right)^{d}$. Este último saldo é visto pela autoridade monetária como o valor necessário do saldo de exportações líquidas para se obter o equilíbrio externo da economia no longo prazo. Dessa forma, a taxa desejada de variação do câmbio real é dada por ${ }^{910}$ :

$$
\dot{\theta}=\beta\left[\frac{E}{K}-\left(\frac{E}{K}\right)^{d}\right]
$$

Onde: $\beta$ representa um parâmetro negativo.

Conforme Neves e Oreiro (2009), vamos admitir que a política monetária é conduzida com base na fixação do valor da taxa nominal de juros. Considerando a existência de inércia inflacionária, sabemos que mudanças na taxa nominal de juros se traduzem, por conseguinte, em mudanças na taxa real de juros.

Nesse contexto, o Banco Central opera em um regime de metas de inflação ${ }^{11}$, calibrando a taxa nominal de juros de maneira a produzir as mudanças no valor da taxa real de juros requeridas para fazer com que a taxa efetiva de inflação convirja com respeito à meta inflacionária. Para compatibilizar a obtenção da meta de inflação com a estabilização do nível de produção, iremos supor que a política monetária funciona com base na seguinte regra de Taylor:

$$
\dot{r}=\lambda_{0}\left(\pi-\pi^{m}\right)+\lambda_{1}\left(u-u^{m}\right)
$$

Onde: $\dot{r}$ é a variação da taxa real de juros com respeito ao tempo; $\lambda_{0}>0$ é a velocidade de reação do Banco Central com respeito às divergências entre a inflação efetiva e a meta de inflação $\left(\pi^{m}\right)$ e $\lambda_{1}<1$ é a velocidade de reação do Banco Central com respeito às divergências entre o grau efetivo de utilização da capacidade

(8) Sobre a capacidade da autoridade monetária de administrar a taxa real de câmbio ver Montiel (2011).

(9) Note que a taxa de variação do câmbio nominal fixada pelo Banco Central será dada por: $\hat{e}=\dot{\theta}+(\pi-$ $\left.\pi^{*}\right)$. Onde $\hat{e}$ é a taxa de variação do câmbio nominal e $\pi^{*}$ é a taxa de inflação internacional.

(10) Como estamos supondo que os trabalhadores não têm poder de mercado (o que implica em uma economia com baixo nível de sindicalização como, por exemplo, a dos Estados Unidos), segue-se que variações da taxa nominal de câmbio não devem dar ensejo a uma aceleração da inflação por conta do fenômeno da real wage resistance.

(11) Deve-se ressaltar que não existe incompatibilidade a priori entre a existência de um regime de metas de inflação e a hipótese de câmbio administrado haja vista que na economia em consideração não existe mobilidade de capitais. 
produtiva e a meta de utilização da capacidade $\left(u^{m}\right)$ definida pelas autoridades monetárias.

\section{Equilíbrio de curto prazo}

No curto prazo tanto a taxa real de câmbio como a taxa real de juros estão dadas. Dessa forma, o equilíbrio macroeconômico é obtido por intermédio de variações do grau de utilização da capacidade produtiva. Temos, então, que:

$$
u=\frac{I}{K}+\frac{C}{K}+\frac{G}{K}+\frac{E}{K}
$$

Desta maneira, ao substituirmos as equações (5), (8), (9) e (10) na equação (15), obtemos o grau de utilização da capacidade produtiva de equilíbrio de curto prazo para a economia em consideração ${ }^{12}$ :

$$
u^{*}=\frac{\left(\alpha_{0}+\varepsilon_{0}+\gamma\right)+\alpha_{1} m(\theta)+\left(\alpha_{3}+\varepsilon_{1}\right) \theta-\alpha_{4} \theta^{2}-\alpha_{5} r}{\left(s_{c} m+\varepsilon_{2}-\alpha_{2}\right)}
$$

Dada a taxa real de juros, o efeito de uma variação na taxa de câmbio real sobre o valor de curto prazo do grau de utilização da capacidade produtiva é expresso por:

$$
\frac{\partial u}{\partial \theta}=\frac{\alpha_{1} m^{\prime}+\left(\alpha_{3}+\varepsilon_{1}\right)-2 \alpha_{4} \theta-u^{*} s_{c} m^{\prime}}{\left(s_{c} m+\varepsilon_{2}-\alpha_{2}\right)}
$$

Antes de comentar o resultado acima, algumas considerações devem ser feitas.

Primeiramente, relembremos a equação (4), a qual mostra que um aumento da taxa de câmbio real gera um aumento da participação dos lucros na renda. Como isto afeta o grau de utilização da capacidade produtiva? Analisando as equações (5) e (8), vemos que uma variação da participação dos lucros na renda tem um efeito ambíguo sobre o grau de utilização da capacidade produtiva. Por um lado, um aumento de $m$ gera um aumento no investimento e, por outro, reduz o consumo agregado, uma vez que a propensão a consumir a partir dos salários é maior do que a propensão a consumir a partir dos lucros.

Podemos afirmar, por conseguinte, que o efeito de uma variação na taxa de câmbio real sobre o grau de utilização da capacidade produtiva também é ambíguo, mas depende do nível da taxa real de câmbio. Dessa forma, uma desvalorização da taxa real de câmbio resultaria em aumento do grau de utilização da capacidade se:

$\theta<\left(\frac{\alpha_{1} m^{\prime}+\alpha_{3}}{2 \alpha_{4}}\right)+\left(\frac{\varepsilon_{1}-u^{*} s_{c} m^{\prime}}{2 \alpha_{4}}\right)$

(12) Iremos supor que $\left(s_{c} m+\varepsilon_{2}-\alpha_{2}\right)>0$, ou seja, que a soma entre a propensão marginal a poupar e a propensão marginal a importar é maior do que a propensão marginal a investir. Essa é uma condição necessária para garantir a estabilidade do equilíbrio de curtoprazo da economia em consideração. 
Ou seja:

$$
\theta<\theta^{*}+C=\theta^{* *}(18 \mathrm{a})
$$

O valor da constante $C$ na equação (19) depende do sinal de $\left(\varepsilon_{1}-u^{*} s_{c} m^{\prime}\right)$. Essa expressão nada mais é do que a diferença entre a sensibilidade das exportações líquidas a uma variação da taxa de câmbio real e a sensibilidade do consumo a uma variação do câmbio. Iremos assumir que essas magnitudes são similares de tal forma que uma desvalorização cambial deverá resultar em uma taxa de substituição da poupança externa (igual ao saldo das exportações líquidas com o sinal trocado) por poupança interna aproximadamente igual a um ${ }^{13}$. Assumindo essa condição como verdadeira temos que o valor crítico da taxa real de câmbio que faz com que a derivada em (17) seja positiva ( $\left.\theta^{* *}\right)$ é igual ao que maximiza a taxa de acumulação de capital $\left(\theta^{*}\right)$. Por conseguinte, se a taxa de câmbio real estiver sobrevalorizada $\left(\theta<\theta^{*}\right)$, então uma depreciação da taxa de câmbio real resultará no aumento da participação dos lucros na renda e no aumento da utilização da capacidade produtiva. Neste caso, o regime de acumulação da economia se caracteriza por ser profit-led. Se, por outro lado, a taxa de câmbio real estiver subvalorizada $\left(\theta>\theta^{*}\right)$, então uma apreciação da taxa de câmbio real resultará em uma redução da participação dos lucros na renda e no aumento da utilização da capacidade produtiva. Neste caso, temos um regime de acumulação do tipo wage-led.

Quadro 1

Regimes de acumulação e taxa de câmbio real

\begin{tabular}{|c|c|c|}
\hline Taxa de Câmbio Real & Efeitos & Regime de Acumulação \\
\hline Sobrevalorizada $\left(\theta<\theta^{*}\right)$ & $\uparrow \theta\left\{\begin{array}{l}\uparrow m \\
\uparrow u\end{array}\right.$ & Profit-Led \\
\hline Subvalorizada $\left(\theta>\theta^{*}\right)$ & $\downarrow \theta\left\{\begin{array}{l}\downarrow m \\
\uparrow u\end{array}\right.$ & Wage-Led \\
\hline
\end{tabular}

Fonte: Elaboração própria.

Dada a taxa de câmbio real, o efeito de uma variação da taxa real de juros sobre o valor de equilíbrio de curto prazo do grau de utilização da capacidade produtiva é expresso por:

$$
\frac{\partial u^{*}}{\partial r}=-\frac{\alpha_{5}}{\left(s_{c} m+\varepsilon_{2}-\alpha_{2}\right)}<0
$$

Na expressão (19) observamos a existência de uma relação inversa entre a taxa real de juros e o grau de utilização da capacidade produtiva.

(13) A respeito do conceito de substituição de poupanças ver Bresser-Pereira (2012, p. 14-16). 
A taxa de inflação de equilíbrio de curto prazo é determinada após substituirmos (16) em (11) e a resultante em (12), obtendo assim a seguinte expressão:

$$
\pi^{*}=\varphi\left[\eta_{0}+\eta_{1} u^{*}(\theta)-m(\theta)\right](20)
$$

Dada a taxa real de juros, o efeito de uma variação da taxa de câmbio real sobre o valor de equilíbrio de curto prazo da taxa de inflação é expresso por:

$$
\frac{\partial \pi^{*}}{\partial \theta}=\varphi\left(\eta_{1} \frac{\partial u}{\partial \theta}-m^{\prime}\right)
$$

Na equação (21) verificamos que o efeito de uma desvalorização cambial sobre a taxa de inflação vai depender do regime de acumulação prevalecente na economia. Se o regime for do tipo wage-led, então $\frac{\partial u}{\partial \theta}<0$ e, portanto, $\frac{\partial \pi^{*}}{\partial \theta}<0$; ou seja, uma desvalorização do câmbio real irá resultar em uma diminuição da taxa de inflação. Contudo, se o regime de acumulação for profit-led, uma desvalorização cambial será seguida por um aumento da taxa de inflação se $\frac{\partial u}{\partial \theta}>\frac{m^{\prime}}{\eta_{1}}=\delta^{*}>0$; ou seja, se a sensibilidade do grau de utilização da capacidade produtiva às variações da taxa real de câmbio for maior do que uma constante positiva $\delta^{*}$.

Dada a taxa de câmbio real, o efeito de uma variação da taxa real de juros sobre o valor de equilíbrio de curto prazo da taxa de inflação é expresso por:

$$
\frac{\partial \pi^{*}}{\partial r}=-\frac{\varphi \eta_{1} \alpha_{5}}{\left(s_{c} m+\varepsilon_{2}-\alpha_{2}\right)}<0
$$

Deste modo, temos que um aumento da taxa real de juros gera uma redução da taxa de inflação.

\section{Dinâmica e equilíbrio de longo prazo}

Iremos supor que, no longo prazo, tanto o grau de utilização da capacidade produtiva como a taxa de inflação correspondem aos seus valores de equilíbrio de curto prazo, como definido pelas equações (16) e (21). A taxa de câmbio real e a taxa real de juros, por outro lado, passam a ser variáveis endógenas, de tal forma que a sua dinâmica é explicada pelas equações (13) e (14):

$$
\begin{gathered}
\dot{\theta}=\beta\left[\frac{E}{K}-\left(\frac{E}{K}\right)^{d}\right](13) \\
\dot{r}=\lambda_{0}\left(\pi-\pi^{m}\right)+\lambda_{1}\left(u-u^{m}\right)(14)
\end{gathered}
$$

A equação (13) nos mostra a dinâmica da taxa real de câmbio a qual depende do hiato entre o saldo efetivo de exportações líquidas e o saldo comercial desejado 
pelo Banco Central. A equação (14), por sua vez, representa a regra de política monetária seguida pelo Banco Central. Sendo assim, variações na taxa real de juros serão induzidas pelo Banco Central sempre que a inflação corrente divergir da meta de inflação de longo prazo e/ou que o grau de utilização da capacidade produtiva for diferente do grau potencial.

Pela expansão da série de Taylor, escrevemos o sistema das equações (13) e (14), da seguinte forma:

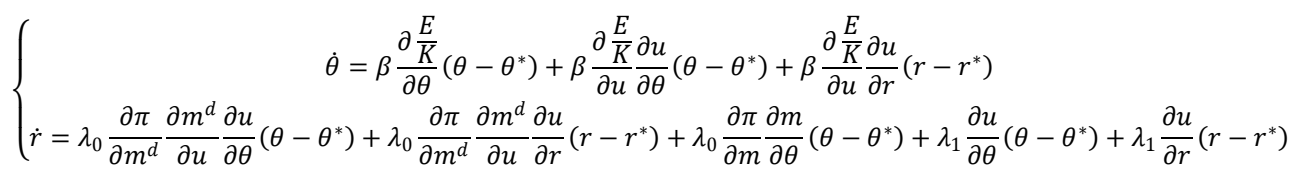

Temos, por conseguinte:

$$
\left[\begin{array}{c}
\dot{\theta} \\
\dot{r}
\end{array}\right]=\left[\begin{array}{cc}
\beta \frac{\partial \frac{E}{K}}{\partial \theta}+\beta \frac{\partial \frac{E}{\partial u} \frac{\partial u}{\partial \theta}}{\partial \pi} & \beta \frac{\partial \frac{E}{K}}{\partial u} \frac{\partial u}{\partial r} \\
\lambda_{0} \frac{\partial \pi}{\partial m^{d}} \frac{\partial m^{d}}{\partial u} \frac{\partial u}{\partial \theta}+\lambda_{0} \frac{\partial m}{\partial m} \frac{\partial u}{\partial \theta}+\lambda_{1} \frac{\partial u}{\partial \theta} & \lambda_{0} \frac{\partial \pi}{\partial m^{d}} \frac{\partial m^{d}}{\partial u} \frac{\partial u}{\partial r}+\lambda_{1} \frac{\partial u}{\partial r} \\
\cdot\left[\begin{array}{l}
\theta-\theta^{*} \\
r-r^{*}
\end{array}\right]
\end{array}\right]
$$

Assim, temos:

$$
\left\{\begin{array}{c}
J_{11}=\beta \frac{\partial \frac{E}{K}}{\partial \theta}+\beta \frac{\partial \frac{E}{K}}{\partial u} \frac{\partial u}{\partial \theta} \\
J_{12}=\beta \frac{\partial \frac{E}{K}}{\partial u} \frac{\partial u}{\partial r} \\
J_{21}=\lambda_{0} \frac{\partial \pi}{\partial m^{d}} \frac{\partial m^{d}}{\partial u} \frac{\partial u}{\partial \theta}+\lambda_{0} \frac{\partial \pi}{\partial m} \frac{\partial m}{\partial \theta}+\lambda_{1} \frac{\partial u}{\partial \theta} \\
J_{22}=\lambda_{0} \frac{\partial \pi}{\partial m^{d}} \frac{\partial m^{d}}{\partial u} \frac{\partial u}{\partial r}+\lambda_{1} \frac{\partial u}{\partial r}
\end{array}\right.
$$

Com base nas características de nossa economia, iremos determinar os sinais dos elementos acima. Em primeiro lugar, sabemos que $\frac{\partial \frac{E}{K}}{\partial u}<0$ e $\frac{\partial u}{\partial r}<0$. Deste modo, temos que $J_{12}>0$. Além disso, como $\frac{\partial \pi}{\partial m^{d}}>0$ e $\frac{\partial m^{d}}{\partial u}>0$, então temos que $J_{22}<0$. Por ora, os sinais de $J_{11}$ e de $J_{21}$ permanecerão indeterminados. Esta questão será aprofundada mais adiante. 
Quadro 2

Análise dos elementos da matriz Jacobiana

\begin{tabular}{|c|c|}
\hline Elemento & Sinal \\
\hline$J_{11}$ & Indeterminado \\
\hline$J_{12}$ & - \\
\hline$J_{21}$ & Indeterminado \\
\hline$J_{22}$ & $\ominus$ \\
\hline
\end{tabular}

Fonte: Elaboração própria.

A análise de estabilidade local do sistema pode ser efetuada calculando o determinante, $\operatorname{det}|J|$, e o traço, $t r|J|$, da matriz Jacobiana. Assim, o sistema será um equilíbrio estável se $\operatorname{det}|J|>0$ e $\operatorname{tr}|J|<0$; instável se $\operatorname{det}|J|>0$ e $\operatorname{tr}|J|>0$; ou será uma trajetória de sela se $\operatorname{det}|J|<0$ e $\operatorname{tr}|J|>0$. O cálculo de $\operatorname{det}|J|$ é dado por:

Isto é:

$$
\operatorname{det}|J|=J_{11} \cdot J_{22}-J_{12} \cdot J_{21}
$$

$$
\begin{aligned}
& \operatorname{det}|J|=\left(\beta \frac{\partial \frac{E}{K}}{\partial \theta}+\beta \frac{\partial \frac{E}{K}}{\partial u} \frac{\partial u}{\partial \theta}\right)\left(\lambda_{0} \frac{\partial \pi}{\partial m^{d}} \frac{\partial m^{d}}{\partial u} \frac{\partial u}{\partial r}+\lambda_{1} \frac{\partial u}{\partial r}\right) \\
& -\left(\beta \frac{\partial \frac{E}{K}}{\partial u} \frac{\partial u}{\partial r}\right)\left(\lambda_{0} \frac{\partial \pi}{\partial m^{d}} \frac{\partial m^{d}}{\partial u} \frac{\partial u}{\partial \theta}+\lambda_{0} \frac{\partial \pi}{\partial m} \frac{\partial m}{\partial \theta}+\lambda_{1} \frac{\partial u}{\partial \theta}\right)
\end{aligned}
$$

Para calcular $\operatorname{tr}|J|$, fazemos:

$$
\operatorname{tr}|J|=J_{11}+J_{22}
$$

Ou seja:

$$
\operatorname{tr}|J|=\beta \frac{\partial \frac{E}{K}}{\partial \theta}+\beta \frac{\partial \frac{E}{K}}{\partial u} \frac{\partial u}{\partial \theta}+\lambda_{0} \frac{\partial \pi}{\partial m^{d}} \frac{\partial m^{d}}{\partial u} \frac{\partial u}{\partial r}+\lambda_{1} \frac{\partial u}{\partial r}
$$

Do exposto acima, podemos ver que a dinâmica de longo prazo irá depender de quais efeitos predominam na economia. Deste modo, é possível identificar quatro situações diferentes:

Quadro 3

Dinâmica de longo prazo

\begin{tabular}{|c|c|c|c|}
\hline & Caso & Determinante & Traço \\
\hline 1 & $J_{11}>0 \mathrm{e} J_{21}<0$ & $\operatorname{det}|J|<0$ & $\begin{array}{l}\operatorname{se} J_{11}>J_{22} \text { então } t r|J|>0 \\
\operatorname{se} J_{11}<J_{22} \text { então } t r|J|<0\end{array}$ \\
\hline 2 & $J_{11}<0 \mathrm{e} J_{21}>0$ & $\operatorname{det}|J|>0$ & $\operatorname{tr}|J|<0$ \\
\hline 3 & $J_{11}>0 \mathrm{e} J_{21}>0$ & $\begin{array}{l}\mathrm{se} J_{11} \cdot J_{22}>J_{12} \cdot J_{21} \text { então } \operatorname{det}|J|<0 \\
\mathrm{se} J_{11} \cdot J_{22}<J_{12} \cdot J_{21} \text { então } \operatorname{det}|J|>0\end{array}$ & $\begin{array}{l}\mathrm{se} J_{11}>J_{22} \text { então } t r|J|>0 \\
\operatorname{se} J_{11}<J_{22} \text { então } t r|J|<0\end{array}$ \\
\hline 4 & $J_{11}<0 \mathrm{e} J_{21}<0$ & $\begin{array}{l}\mathrm{se} J_{11} \cdot J_{22}>J_{12} \cdot J_{21} \text { então det }|J|>0 \\
\mathrm{se} J_{11} \cdot J_{22}<J_{12} \cdot J_{21} \text { então det }|J|<0\end{array}$ & $\operatorname{tr}|J|<0$ \\
\hline
\end{tabular}

Fonte: Elaboração própria. 
Uma variação na taxa de câmbio real pode afetar a economia em questão de duas maneiras distintas. Pelo lado da demanda agregada, temos que um aumento da taxa de câmbio real gera um aumento das exportações líquidas. O lado da oferta, por sua vez, é afetado na medida em que um aumento da taxa de câmbio real gera aumento do mark-up das firmas, via aumento do poder de monopólio. No entanto, dadas as características de nossa economia, o efeito renda não pode ser maior do que o efeito substituição, pois isto neutralizaria os ganhos de competitividade obtidos com a desvalorização cambial. Desta maneira, iremos supor que o efeito de uma variação da taxa de câmbio real sobre as exportações líquidas é maior do que o efeito de uma variação da taxa de câmbio real sobre a utilização da capacidade produtiva que, por sua vez, é maior do que o efeito de uma variação da taxa de câmbio real sobre a taxa de mark-up. Em resumo, temos que $\frac{\partial \frac{E}{K}}{\partial \theta}>\frac{\partial u}{\partial \theta}>\frac{\partial m}{\partial \theta}$. Como $\frac{\partial m}{\partial \theta}>0$, segue-se que nas condições supostas o regime de acumulação é do tipo profit-led. Temos, então, que:

Quadro 4

Análise dos elementos da matriz Jacobiana *

\begin{tabular}{|c|c|}
\hline Elemento & Sinal \\
\hline$J_{11}$ & - \\
\hline$J_{12}$ & - \\
\hline$J_{21}$ & $\oplus$ \\
\hline$J_{22}$ & $\ominus$ \\
\hline
\end{tabular}

Nota: (*) Supondo que o regime de acumulação é do tipo profit-led.

Fonte: Elaboração própria.

Podemos descartar, portanto, a ocorrência dos casos 1, 3 e 4. Desta maneira, a dinâmica de longo prazo da economia se caracteriza por apresentar um equilíbrio estável.

A partir do exposto acima, estamos aptos para determinar as inclinações dos lócus $\dot{\theta}=0$ e do lócus $\dot{r}=0$, ou seja, as combinações de $\theta$ e $r$ para as quais a taxa de câmbio real e a taxa real de juros permanecem constantes ao longo do tempo. Fazendo $\dot{\theta}=0$ e $\dot{r}=0$, obtemos:

$$
\begin{gathered}
\left.\frac{\partial \theta}{\partial r}\right|_{\dot{\theta}=0}=-\frac{\beta \frac{\partial \frac{E}{K}}{\partial u} \frac{\partial u}{\partial r}}{\beta \frac{\partial \frac{E}{K}}{\partial \theta}+\beta \frac{\partial \frac{E}{K}}{\partial u} \frac{\partial u}{\partial \theta}}=-\frac{J_{12}}{J_{11}} \\
\left.\frac{\partial \theta}{\partial r}\right|_{\dot{r}=0}=-\frac{\lambda_{0} \frac{\partial \pi}{\partial m^{d}} \frac{\partial m^{d}}{\partial u} \frac{\partial u}{\partial r}+\lambda_{1} \frac{\partial u}{\partial r}}{\lambda_{0} \frac{\partial \pi}{\partial m^{d}} \frac{\partial m^{d}}{\partial u} \frac{\partial u}{\partial \theta}+\lambda_{0} \frac{\partial \pi}{\partial m} \frac{\partial m}{\partial \theta}+\lambda_{1} \frac{\partial u}{\partial \theta}}=-\frac{J_{22}}{J_{21}}
\end{gathered}
$$


Temos, portanto, que a inclinação do lócus $\dot{\theta}=0$ é negativa, e a inclinação do lócus $\dot{r}=0$ é positiva. Dessa forma, a configuração de equilíbrio de longo prazo do sistema pode ser visualizada na Figura 2 abaixo:

Figura 2

Equilíbrio de Longo Prazo do Sistema*

$\Theta$

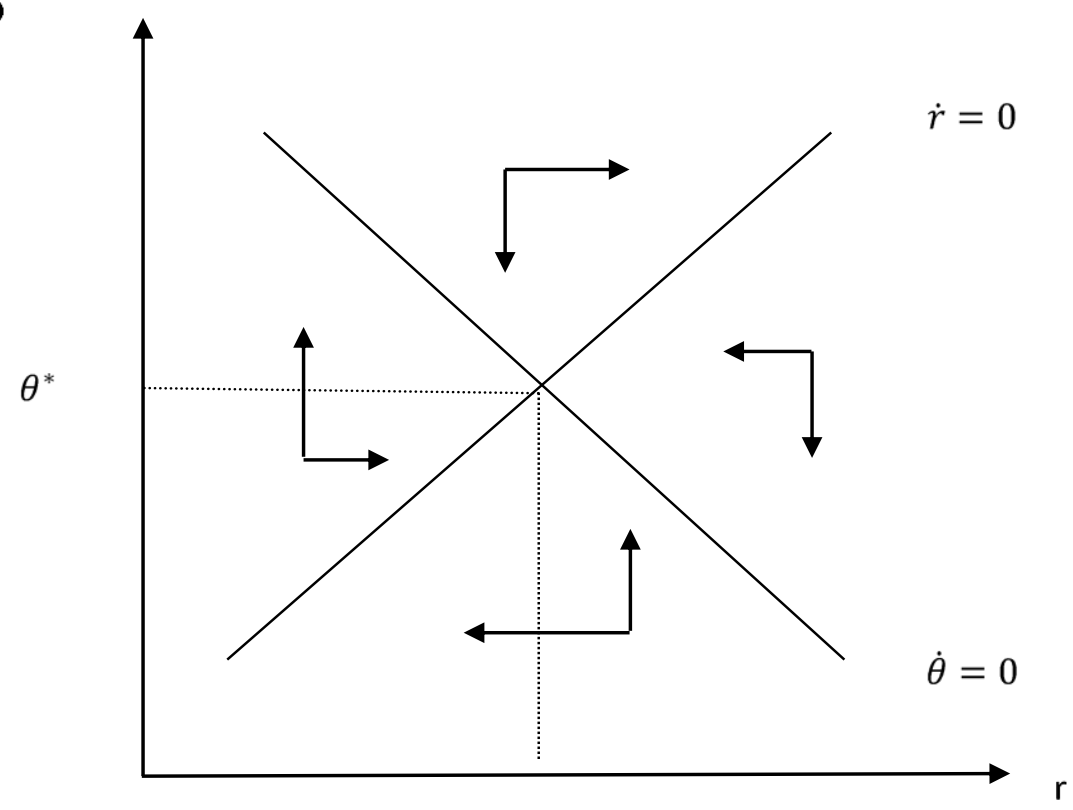

(*) Supondo um regime de acumulação do tipo profit-led.

Uma vez que definimos as condições de estabilidade do sistema, podemos realizar um exercício de estática comparativa para avaliar o efeito sobre o equilíbrio de longo prazo do sistema de uma elevação da meta de inflação.

Se o Banco Central aumentar a meta de inflação, então o lócus $\dot{r}=0$ se deslocará para cima e para a esquerda, como observado na Figura 3. Dessa forma, após um período inicial de ajuste, a economia irá convergir para uma nova posição de equilíbrio de longo prazo caracterizada por uma taxa real de juros mais baixa e uma taxa real de câmbio mais depreciada. A combinação entre juro real mais baixo e câmbio mais depreciado deverá levar a um aumento do grau de utilização da capacidade produtiva, à medida que a economia opera sob um regime profit-led. Os efeitos combinados da taxa real de câmbio mais desvalorizada, do juro real mais baixo e do nível mais alto de utilização da capacidade produtiva irão induzir um aumento da taxa de acumulação de capital, de forma a acelerar o crescimento econômico de longo prazo. Do ponto de vista da distribuição funcional da renda, contudo, a desvalorização do câmbio real irá permitir um aumento da taxa de markup e, portanto, um aumento (diminuição) da participação dos lucros (dos salários) na 
renda nacional. Além disso, o aumento do grau de utilização da capacidade produtiva resultante da desvalorização da taxa real de câmbio levou as firmas a elevar o markup desejado e, portanto, a participação desejada dos lucros na renda, resultando assim em uma aceleração da taxa de inflação. Daqui se segue, portanto, que o modelo aqui apresentado prevê a existência tanto de um trade-off entre crescimento e distribuição de renda a longo prazo, como de um trade-off entre câmbio e inflação no longo prazo.

Figura 3

Efeitos de longo prazo um aumento da meta de inflação

$\Theta$

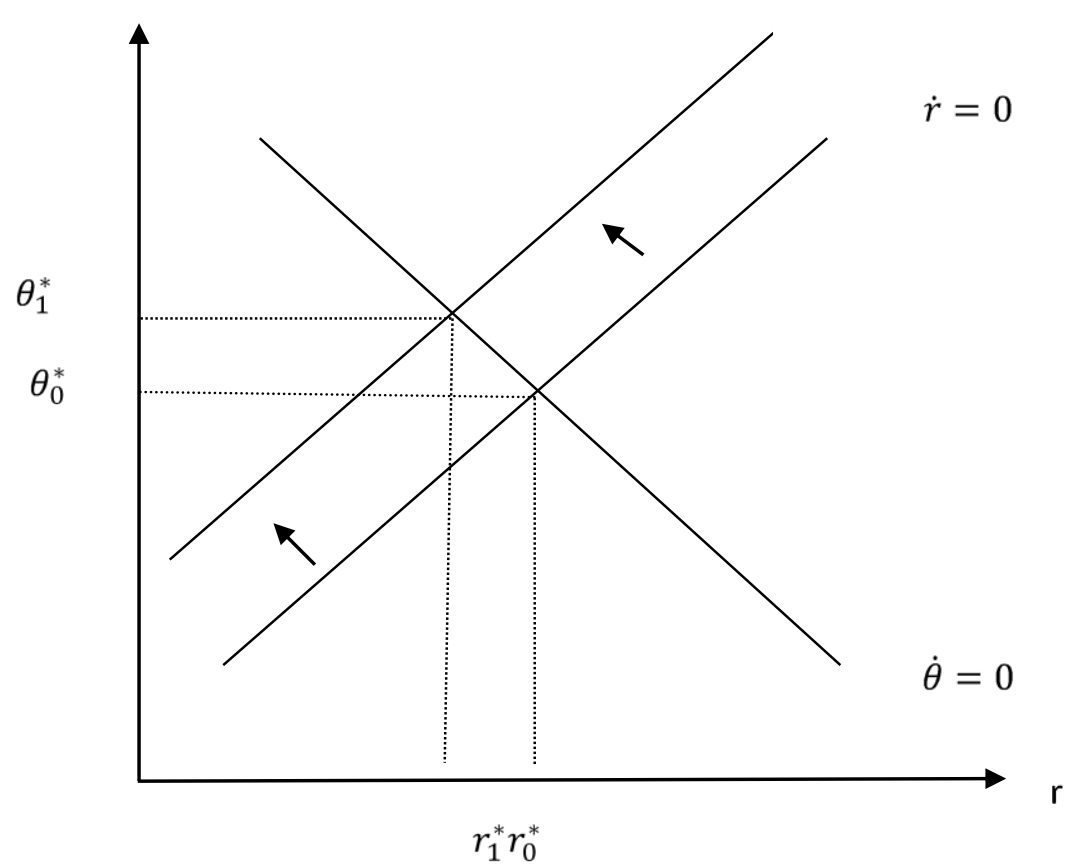

\section{Comentários finais}

Neste artigo foi desenvolvido um modelo teórico pós-keynesiano de crescimento e distribuição que engloba aspectos fundamentais para o entendimento das economias modernas, notadamente a administração da taxa de câmbio real e o processo de fixação da taxa de juros pelo Banco Central. O modelo aqui desenvolvido foi uma extensão do modelo de Oreiro e Araújo (2013) na qual se incluiu um processo inflacionário decorrente da tentativa das firmas de ajustar o mark-up efetivo ao mark-up desejado, um regime de câmbio administrado no qual a autoridade monetária procura fazer um ajuste gradual da taxa real de câmbio de forma a obter o equilíbrio externo de longo prazo da economia, e uma regra de Taylor para a política monetária. 
Na sequência se discute as propriedades do equilíbrio de curto e de longo prazo do modelo. No equilíbrio de curto prazo verifica-se que a relação entre crescimento e distribuição, o assim chamado regime de acumulação, depende da relação entre a taxa real de câmbio e o valor ótimo dessa taxa. Quando a taxa real de câmbio está sobrevalorizada, ou seja, abaixo do valor ótimo, o regime de acumulação é profit-led. Quando a taxa real de câmbio se encontra subvalorizada, ou seja, acima do seu valor ótimo, então o regime de acumulação é do tipo profit-led.

No equilíbrio de longo prazo verifica-se que um aumento da meta de inflação está associado a uma redução da taxa real de juros e a um aumento da taxa real de câmbio, o que resulta em uma elevação da taxa de acumulação de capital. Contudo, o aumento da taxa de mark-up viabilizado pela desvalorização cambial levará a um aumento (redução) da participação dos lucros (salários) na renda, bem como a um aumento da taxa de inflação; de maneira que observamos a existência tanto de um trade-off entre crescimento e distribuição de renda, como entre câmbio e inflação no longo prazo.

\section{Referências bibliográficas}

ARAUJO, E., GALA, P. Regimes de crescimento econômico no Brasil: evidências empíricas e implicações de política. Estudos Avançados, v. 25, n. 75, 2012.

BASILIO, F.; OREIRO, J. L. Crescimento endógeno, conflito distributivo e política monetária: um modelo pós-keynesiano com fluxos de capitais para uma pequena economia aberta. In: ENCONTRO DE ECONOMIA DA REGIÃO SUL, 11, Cuririba, 2008. Anais...

BHADURI, A.; MARGLIN, S. Unemployment and the real wage: the economic basis for contesting political ideologies. Cambridge Journal of Economics, v. 14, p. 375-393, 1990.

BLANCHARD, O. Macroeconomia. 4. ed. São Paulo: Pearson. 2007.

BLECKER, R. A. International competition, income distribution and economic growth. Cambridge Journal of Economics, v. 32, p. 273-288, 1989.

BLECKER, R. A. Open economy models of distribution and growth. in: HEIN, E., STOCKHAMMER, E. (Ed.). A modern guide to Keynesian macroeconomics and economic policies. Aldesrhot: Edward Elgar, 2010.

BRESSER-PEREIRA, L. C. A taxa de câmbio no centro da teoria do desenvolvimento. EstudosAvançados, v. 26, n. 75, 2012.

EICHENGREEN, B. The real exchange rate and economic growth. Washington, DC: World Bank. Commission on Growth and Development, 2008. 
FRENKEL, R. Real exchange rate and employment in Argentina, Brazil, Chile and Mexico. Caba, Argentina: Centro de Estudios de Estado y Sociedad, 2004.

HEIN, E.; VOGEL, L. Distribution and growth reconsidered: empirical results for six OECD countries. Cambridge Journal of Economics, v. 8, n. 1, p. 25-40, 2008.

KALDOR, N. Alternative theories of distribution. Review of Economic Studies, v. 33, p. 83-100, 1956.

McCOMBIE, J. S. L.; ROBERTS, M. The role of the balance of payments in economic growth. In: SETTERFIELD, M. (Org.). The economics of demand ledgrowth. Aldershot: Edward Elgar, 2002.

MONTIEL, P. Macroeconomics in emerging markets. Cambridge: Cambridge University Press, 2011.

NEVES, A. L.; OREIRO, J. L. Crescimento, distribuição de renda e metas de inflação num modelo macrodinâmico pós-keynesiano. Revista Economia, v. 10, n. 3, 2009.

OREIRO, J. L.; PUNZO; L.; ARAUJO, E. Macroeconomic constraints to growth of Brazilian economy: diagnosis and some policy proposals. Cambridge Journal of Economics, v. 36, p. 919-939, 2012.

OREIRO, J. L.; ARAUJO, E. Exchange rate misalignment, capital accumulation and income distribution: theory and evidence from the case of Brazil. Panoeconomicus, v. 3, Special Issue, p. 381-396, 2013.

RAZIN, O.; COLLINS, S. Real exchange rate misalignments and growth. Cambridge: NBER, 1997. (Working Paper, n. 6147).

RODRIK, D. Real exchange rate and economic growth: theory and evidence. Cambridge, MA: John F. Kennedy School of Government, Harvard University, Draft, Jul. 2008.

THIRLWALL, A. P. The balance of payments constraint as an explanation of international growth rate differences. Banca Nazional e del Lavoro Quarterly Review, n. 128, Mar. 1979. 